\title{
High body mass index and night shift work are associated with COVID-19 in health care workers
}

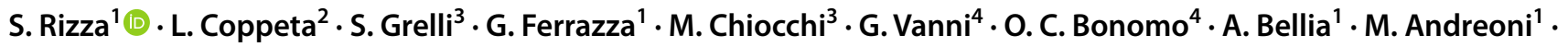 \\ A. Magrini ${ }^{2} \cdot$ M. Federici ${ }^{1}$
}

Received: 9 July 2020 / Accepted: 15 August 2020 / Published online: 27 August 2020

(C) Italian Society of Endocrinology (SIE) 2020

\begin{abstract}
Objective To assess the magnitude of COVID-19 spread and the associated risk factors among health care workers (HCWs), we conducted an in-hospital survey in a central Italian COVID Hospital.

Methods Participants underwent nasopharyngeal swab and/or serum collection for SARS-CoV-2 IgG examination. We divided participants according to working status, into rotating-night shift workers (r-NSW) and day-workers.

Results We found 30 cases of COVID-19 infection in a total of 1180 HCWs (2.5\%). Most COVID-19-positive hospital employees were r-NSWs with significantly higher BMI than that of individuals who tested negative. After adjustment for covariates, night work and BMI > 30 were associated with a markedly greater risk of COVID-19 diagnosis (OR 3.049 [95\% CI 1.260-7.380] and OR 7.15 [95\%CI 2.91-17.51], respectively).

Conclusions Our results describe a low prevalence of COVID-19 infection among HCWs at a central Italian COVID Hospital. COVID-19 infection risk appears to be associated with obesity and night shift work, thus supporting the need for careful health surveillance among frontline HCWs exposed to COVID-19.
\end{abstract}

Keywords SARS-CoV-2 $\cdot$ COVID-19 $\cdot$ Night shift work $\cdot$ Obesity $\cdot$ Health care worker

\section{Introduction}

Since the first case of COVID-19 in Italy was reported on February 28, 2020, the number of infected patients has grown exponentially [1]. However, the infection spread in Italy was more pronounced in northern Italy (Lombardy, Piedmont, Liguria, and Veneto) than in all other Italian areas. Central-southern Italian regions had fewer cases of

S. Rizza and L. Coppeta have contributed equally to the manuscript.

S. Rizza

stefano.rizza@uniroma2.it

1 Department of Systems Medicine, University of Rome Tor Vergata, Via Montpellier 1, 00133 Rome, Italy

2 Department of Biomedicine and Prevention, University of Rome Tor Vergata, Rome, Italy

3 Department of Experimental Medicine, University of Rome Tor Vergata, Rome, Italy

4 Department of Surgical Science, University of Rome Tor Vergata, Rome, Italy
COVID-19 and consequent deaths. As of the beginning of April, 2020, the WHO reported 22,073 cases of COVID-19 among health care workers (HCWs) from 52 countries [2]. It is now clear that COVID-19 has strong infectivity and a high transmission speed, and can cause serious infections among HCWs. Although the risk factors for unfavorable outcomes including aging, male sex, and underlying comorbiditiessuch as obesity, hypertension, diabetes, and cardiovascular disease-have been extensively reported, uncertainty remains regarding the clinical risk factors for COVID-19 infection, particularly among frontline HCWs exposed to COVID-19 infection. To address this a lack of knowledge, here, we report the preliminary results of a COVID-19 outbreak investigation in a central Italian COVID hospital (Tor Vergata University hospital).

\section{Materials and methods}

The local ethics committee approved the study, and written informed consent was provided by all survey participants before their enrollment. The survey was anonymous, and 
the confidentiality of information was assured. The study was a cross-sectional, hospital-based survey conducted via the Tor Vergata occupational medicine service from March 13, 2020 to May 5, 2020. Among the 1912 health care hospital employees at the time, the study included 1,180 participants (457 [38.7\%] physicians and 723 [61.3\%] nurses). We divided participants into the following groups according to working status: (1) rotating night shift workers (r-NSW) $(n=667)$ working a shift schedule of two-to-seven $12 \mathrm{~h}$ nights per month followed by 2 days off, and (2) day-workers $(n=513)$ who had never worked night shifts. We also grouped participants according to their type of work (physicians and nurses). Finally, we defined HCWs as being at high risk of COVID-19 infection if they worked in departments at the frontline of diagnosis, treatment, and care of patients with COVID-19 [high-risk departments (infectious disease, pneumology, internal medicine, and emergency room) vs low-risk departments (others)]. Data collection was performed by the Service of Occupational Medicine of University of Rome Tor Vergata. Collected data included clinical and demographic characteristics [age, sex, BMI (body mass index), and history of smoking], and comorbidities such as hypertension, diabetes, hyperlipidemia, coronary artery disease (CAD), heart failure, cerebrovascular disease, chronic obstructive pulmonary disease (COPD), active and previous malignancy, chronic kidney disease (CKD) [eGFR equal to or lower than $60 \mathrm{ml} \mathrm{min}^{-1}(1.73 \mathrm{~m})^{-2}$, using the Chronic Kidney Disease Epidemiology Collaboration (CKD-EPI) formula], and pertinent home medications (ace inhibitors and/or angiotensin II receptor blockers). Participants underwent nasopharyngeal swab collection for SARS-CoV-2 realtime reverse transcriptase-polymerase chain reaction testing (AllpexTM 2019-nCOV Assay). Nasopharyngeal samples were tested within a few hours after collection and without any cooling or freezing step. Estimates of the sensitivity $(74 \%$; 95\% CI 68-80\%) and specificity $(99.7 \%$; $95 \%$ CI 99.5-99.9\%) were obtained from 49 healthy control patients and 52 confirmed by PCR cases of COVID-19. We also performed a serum collection for SARS-CoV-2 IgG examination using the LIAISON SARS-CoV-2 S1/S2 IgG test with a high sensitivity (97.4\%) and specificity (98.5\%).

\section{Statistical analysis}

Quantitative data are expressed as mean $\pm \mathrm{SD}$, whereas categorical variables are expressed as number (percentage) of participants. As described above, the main objective of the study was to perform a descriptive investigation. Univariate logistic regression models were used to calculate odds ratios associated with COVID-19-positive tests. In our initial statistical analysis plan, coexistent disorders, age, sex, smoking status, and type of hospital work were systematically forced in the models. However, because most comorbidities, smoking status, and type of hospital work did not contribute to the risk of the primary outcome, our multivariable models ultimately took only age and sex into account. Other variables were considered only if they were associated with the main outcome in univariate analysis and were selected in the final model. All $p$ values are two-tailed, and the significance level was fixed at $5 \%$ before the analysis. All analyses were performed in SPSS version 19.0 for Windows.

\section{Results}

We found only 30 cases of COVID-19 infection ( $n=24$ nasopharyngeal swab and $n=6$ IgG positive), 25 either asymptomatic or with mild symptoms compared with 5 individuals with severe clinical manifestations. The prevalence rate is $2.5 \%$ and the weekly incidence rate is 3.3 COVID-19 cases per 1000 individuals. Most COVID-19 hospital employees were r-NSWs with significantly higher BMI, whereas the groups with and without COVID-19 did not differ with respect to age, current smoking status, and sex. Of note, the risk of in-hospital COVID-19 transmission was unrelated to the type of work (nurses/physicians) or the clinical care environment (high- vs low-risk departments) (Table 1). Hypertension was more frequent in COVID-19 infection cases whereas the distribution of other comorbidities was similar between the two groups. Moreover, treatment with renin-angiotensin-aldosterone system (RAAS) blockers [ACE inhibitors and/or angiotensin II receptor blockers (ARBs)] was used by $6.6 \%$ of individuals with and $0.8 \%$ of individuals without COVID-19. In a regression model adjusted for age, sex, and BMI $>30$, hypertension and night shift work were significantly associated with in-hospital COVID-19 infection, independently of other study covariates (Table 2). In particular, obesity, but not overweight, was associated with a markedly greater risk of COVID-19 diagnosis than that of normal-weight HCWs, with a 7.15 estimated odds ratio (95\% CI 2.91-17.51). Interestingly, NSW and hypertension remained significantly associated with the study outcome, even after adjustment (OR 3.049, 95\% CI 1.260-7.380 and OR 4.392, 95\% CI 1.517-12.718, respectively).

\section{Discussion}

Our preliminary results suggest that r-NSWs are at greater risk of COVID-19 infection risk, thus indicating another possible negative health consequence associated with shift work $[3,4]$, which, in the health care sector, accounts for approximately $45 \%$ of workers. Despite its economic and social relevance, shift work results in a disruption of biological rhythms, thus predisposing individuals to poor 
Table 1 Main characteristics of the HCW population according to SARS-CoV-2 testing results

\begin{tabular}{|c|c|c|c|c|c|}
\hline & \multirow{2}{*}{$\begin{array}{l}\text { Overall population } \\
(n=1180)\end{array}$} & \multicolumn{2}{|l|}{ Study outcome } & \multicolumn{2}{|l|}{ Disease severity } \\
\hline & & $\begin{array}{l}\text { No COVID-19 } \\
(n=1150)\end{array}$ & COVID-19 $(n=30)$ & $\begin{array}{l}\text { Non-severe or asymp- } \\
\text { tomatic }(n=25)\end{array}$ & Severe $(n=5)$ \\
\hline Age (years) & $42.5 \pm 9.5$ & $42.4 \pm 9.3$ & $44.7 \pm 9.3$ & $42.0 \pm 9.5$ & $53.6 \pm 7.5$ \\
\hline Female sex $(n, \%)$ & $768(65.0)$ & $750(65.2)$ & $18(60)$ & $8(32)$ & $2(40)$ \\
\hline Current smoker $(n, \%)$ & $357(30.2)$ & $349(29.9)$ & $8(26.7)$ & $6(24)$ & $2(40)$ \\
\hline $\mathrm{BMI}\left(\mathrm{kg} / \mathrm{m}^{2}\right)$ & $23.8 \pm 3.9$ & $23.7 \pm 3.8$ & $26.9 \pm 4.6$ & $26.6 \pm 4.9$ & $26.9 \pm 4.2$ \\
\hline r-NSWs $(n, \%)$ & $666(56.4)$ & $644(56)$ & $23(76.7)$ & $20(80)$ & $3(60)$ \\
\hline Physicians $(n, \%)$ & $457(38.7)$ & $444(38.6)$ & $13(43.3)$ & $10(40)$ & $3(60)$ \\
\hline High-risk department HCWs $(n, \%)$ & $388(32.8)$ & $379(32.9)$ & $9(30)$ & $10(40)$ & $1(20)$ \\
\hline \multicolumn{6}{|l|}{ Coexisting disorders $(n, \%)$} \\
\hline Any & $121(10.2)$ & $112(9.7)$ & $9(30)$ & $7(28)$ & $2(66.6)$ \\
\hline Hypertension & $55(4.6)$ & $49(4.3)$ & $6(20)$ & $4(16)$ & $2(66.6)$ \\
\hline Diabetes & $27(2.3)$ & $26(2.3)$ & $1(3.3)$ & $1(4)$ & $0(0)$ \\
\hline Hyperlipidemia & $33(2.8)$ & $31(2.7)$ & $2(6.6)$ & $2(8)$ & $0(0)$ \\
\hline Coronary artery disease & $20(1.7)$ & $20(1.7)$ & $0(0)$ & $0(0)$ & $0(0)$ \\
\hline Cerebrovascular disease & $14(1.2)$ & $13(1.1)$ & $1(3.3)$ & $1(4)$ & $0(0)$ \\
\hline Heart failure & $10(0.8)$ & $10(0.8)$ & $0(0)$ & $0(0)$ & $0(0)$ \\
\hline Asthma & $16(1.3)$ & $13(1.1)$ & $3(10)$ & $2(8)$ & $1(20)$ \\
\hline COPD & $12(10.1)$ & $12(1.0)$ & $0(0)$ & $0(0)$ & $0(0)$ \\
\hline Chronic renal disease & $11(9.3)$ & $11(0.9)$ & $0(0)$ & $0(0)$ & $0(0)$ \\
\hline Active malignancy & $2(0.2)$ & $2(0.2)$ & $0(0)$ & $0(0)$ & $0(0)$ \\
\hline Previous malignancy & $15(1.3)$ & $15(1.3)$ & $0(0)$ & $0(0)$ & $0(0)$ \\
\hline Chronic liver disease & $11(9.3)$ & $10(0.8)$ & $1(3.3)$ & $1(4)$ & $0(0)$ \\
\hline ACEi or ARB use $(n,(\%)$ & $37(3.1)$ & $34(0.8)$ & $3(10.0)$ & $2(8)$ & $1(20)$ \\
\hline
\end{tabular}

$B M I$ body mass index, $r$-NSWs rotating night shift workers, $H C W s$ health care workers, $C O P D$ chronic obstructive pulmonary disease, $A C E i$ angiotensin-converting enzyme inhibitors, $A R B$ angiotensin receptor blockers

metabolic health by promoting excess caloric intake and/or decreasing energy expenditure in response to reduced sleep [5]. Notably, obesity is increasingly being described as an underappreciated risk factor for COVID-19 [6], and some studies have recently reported that, in overweight people with COPD, compared with individuals who are not overweight, elevated ACE2 expression may be associated with a greater rate of SARS-CoV-2 infection of the respiratory tract [7]. This point is particularly relevant, because the prevalence of obesity is approximately $40 \%$ in the USA, $6.2 \%$ in China, 20\% in Italy, and 24\% in Spain, all of which have seen substantial COVID-19 spread [8]. Obesity, because it is a pro-inflammatory state, may increase oxidative stress and the risk of type 2 diabetes; it may also restrict ventilation by hindering diaphragm excursion [9]. Whether the relationship between night shift work and in-hospital spread of SARS-CoV-2 might be mediated by low-grade inflammation associated with greater adiposity, which, in turn, could also impair the immune response to viral infection, remains to be determined [10]. Moreover, this relationship might also be an effect of a more intensive workload during night shifts. Alternately, since lung expression of ACE2 will also manifest circadian changes through cell-autonomous regulations and indirect effects of circadian changes in the renin-angiotensin system, it is conceivable the rhythmic expression of clock genes (Per2, Rev-ERB $\alpha$, and BMAL1), frequently disrupted in sleep disturbances, may be involved in SARS-Cov-2 infection [11]. As previously reported, our results depict hypertension as the most prevalent comorbidity in the adult population with COVID-19 [12]. In addition, RAAS blockers were not independently associated with COVID-19 infection, supporting the recent recommendation not to discontinue RAAS blockade. When the other study variables were taken into account, our results did not support an independent association between COVID-19 diagnosis and several medical disorders such as diabetes, coronary artery disease, cerebrovascular disease, COPD, chronic liver disease, chronic renal disease and malignancies as well as age, sex, type of hospital work, or clinical care environment.

Our work has several limitations. First, our study mainly used a cross-sectional approach, and no prospective analysis has been performed to date. Second, we were unable to take into account individual characteristics that might affect obesity or circadian oscillation of the 
Table 2 Univariate and multivariate analyses of the study outcome

\begin{tabular}{llrlr}
\hline & Univariate OR (95\% CI) & \multicolumn{1}{l}{ M } & Multivariate OR (95\% CI) & $p^{*}$ \\
\hline Age & $1.020(0.983-1.059)$ & 0.299 & $1.006(0.965-1.048)$ & 0.779 \\
Female sex & $1.250(0.596-2.621)$ & 0.921 & $1.195(0.555-2.573)$ & 0.649 \\
Current smoker & $0.720(0.317-1.634)$ & 0.432 & & \\
BMI class & 1 & & & \\
$\quad<25$ & $1.358(0.537-3.435)$ & 0.519 & $1.249(0.481-3.246)$ & 0.816 \\
25-29.99 & $7.783(3.302-18.346)$ & $<0.001$ & $7.146(2.916-17.513)$ & $<0.001$ \\
$\geq 30$ & $2.571(1.095-6.041)$ & 0.030 & $3.049(1.260-7.380)$ & 0.013 \\
r-NSWs & $0.795(0.383-1.652)$ & 0.539 & & \\
Physicians & $0.804(0.379-1.705)$ & 0.569 & & \\
High-risk department HCWs & $3.972(1.776-8.883)$ & 0.001 & & \\
Any coexisting disorders & $5.617(2.196-14.370)$ & $<0.001$ & $4.392(1.517-12.718)$ & \\
Hypertension & $1.491(0.196-11.362)$ & 0.700 & & \\
Diabetes & $2.576(0.587-11-297)$ & 0.210 & & \\
Hyperlipidemia & $0.000(0.000-0.000)$ & 0.998 & & \\
Coronary artery disease & $3.016(0.382-23.831)$ & 0.295 & & \\
Cerebrovascular disease & $0.000(0.000-0.000)$ & 0.999 & & \\
Heart failure & $0.000(0.000-0.000)$ & 0.999 & \\
COPD & $0.000(0.000-0.000)$ & 0.999 & \\
Chronic renal disease & $0.000(0.000-0.000)$ & 0.999 & \\
Active malignancy & $0.000(0.000-0.000)$ & 0.999 & & \\
Previous malignancy & $3.931(0.487-31.731)$ & 0.199 & & \\
Chronic liver disease & $3.119(0.971-12.611)$ & 0.098 & & \\
ACEi or ARB use & & & \\
\hline & & & \\
\end{tabular}

$B M I$ body mass index, $r$-NSWs rotating-night shift workers, $H C W s$ health care workers, $C O P D$ chronic obstructive pulmonary disease, $A C E i$ angiotensin-converting enzyme inhibitors, $A R B$ angiotensin receptor blockers

*Age- and sex-adjusted odds ratio endogenous clock, such as total working hours, leisure activities (indoor or outdoor), or diet. Third, the number of participants was relatively small and not balanced between the groups. Fourth, although family exposure remains a possible method of COVID-19 transmission, in particular because Italy was in lockdown at the time of the survey and leisure activity was limited, we do not have information to date about possible exposure outside the workplace.

However, some aspects of this study are noteworthy. To the best of our knowledge, this is the first report of a significant association between obesity, night shift work, and COVID-19 infection in relatively young hospital workers. Whether this association might also be present in NSWs outside the health care system should be assessed, because maintaining greater vigilance for at-risk individuals might decrease the prevalence of severe COVID-19 disease. Finally, we found a particularly low prevalence of COVID-19 infection among HCWs in a central Italian COVID hospital; this result might be an effect of the lockdown concomitant with the increase in COVID-19 infections in the Lazio region.

\section{Conclusions}

In this survey, we report a low prevalence of COVID-19 in-hospital spread in central-southern Italy, but a significant association between infection and obesity and night shift work. Special interventions to promote health surveillance among frontline HCWs exposed to COVID-19 must be immediately implemented. Our results are particularly relevant for public health and policy strategies to mitigate the ongoing spread of COVID-19, and for future approaches to address a possible resurgence of the disease with the imminent lockdown exit in Italy.

Acknowledgements M.F.'s laboratory was funded in part by University (MIUR) Progetti di Ricerca di Interesse Nazionale (PRIN; protocol numbers 2015MPESJS_004 and 2017FM74HK) and EU-FP7 EURHYTHDIA (Grant agreement ID: 278397).

Author contributions SR and LC designed the study. SR, GV, AB., $\mathrm{MC}, \mathrm{OCB}, \mathrm{LC}$, and GF collected clinical information. SG performed real-time reverse transcriptase-polymerase chain testing and $\mathrm{IgG}$ examination for SARS-CoV-2. SR performed statistical analysis and 
drafted the manuscript. MF, AM, and MA revised the final full text. MF provided funding. SR is the guarantor of this work.

\section{Compliance with ethical standards}

Conflict of interest All authors declare that they have no personal or financial conflicts of interest.

Ethical approval All procedures performed in this work involving human participants were in accordance with ethical standards of the institutional research committee and with the 1964 Helsinki declaration and its later amendments or comparable ethical standards.

Informed consent Written informed consent was provided by all survey participants before their enrollment.

\section{References}

1. Lipsitch M, Swerdlow DL, Finelli L (2020) Defining the Epidemiology of Covid-19 - Studies Needed. N Engl J Med 382(13):11941196. https://doi.org/10.1056/NEJMp2002125

2. World Health Organization (2020) Coronavirus Disease 2019 (COVID-19) Situation Report-82, 11 April 2020 World Health Organization, Geneva, Switzerland. https://www.who.int/docs/ default-source/coronaviruse/situation-reports/20200411-sitre p-82-covid-19.pdf?sfvrsn=74a5d15_2. Accessed 11 Apr 2020

3. Rizza S, Pietroiusti A, Farcomeni A et al (2020) Monthly fluctuations in 25-hydroxy-vitamin D levels in day and rotating night shift hospital workers. J Endocrinol Invest. https://doi. org/10.1007/s40618-020-01265-x

4. Rizza S, Neri A, Capanna A et al (2020) Night shift working is associated with an increased risk of thyroid nodules. J Occup
Environ Med 62(1):1-3. https://doi.org/10.1097/JOM.00000 00000001711

5. Zoto E, Cenko F, Doci P, Rizza S (2019) Effect of night shift work on risk of diabetes in healthy nurses in Albania. Acta Diabetol 56(7):811-813. https://doi.org/10.1007/s00592-019-01307-8

6. Kass DA, Duggal P, Cingolani O (2020) Obesity could shift severe COVID-19 disease to younger ages. Lancet 395(10236):1544 1545. https://doi.org/10.1016/S0140-6736(20)31024-2

7. Higham A, Singh D (2020) Increased ACE2 expression in the bronchial epithelium of COPD patients who are overweight. Obesity (Silver Spring). https://doi.org/10.1002/oby.22907

8. GBD 2015 Obesity Collaborators, Afshin A, Forouzanfar MH et al (2017) Health effects of overweight and obesity in 195 countries over 25 years. N Engl J Med 377:13-27

9. Arany Z (2019) Taking a BAT to the chains of diabetes. N Engl J Med 381(23):2270-2272. https://doi.org/10.1056/NEJMcibr19 11353

10. Honce R, Schultz-Cherry S (2019) Impact of obesity on influenza A virus pathogenesis, immune response, and evolution. Front Immunol 10:1071

11. Meira E, Cruz M, Miyazawa M, Gozal D (2020) Putative contributions of circadian clock and sleep in the context of SARSCoV-2 infection. Eur Respir J. 55(6):2001023. https://doi. org/10.1183/13993003.01023-2020

12. Zhou F, Yu T, Du R, Fan G, Liu Y, Liu Z et al (2020) Clinical course and risk factors for mortality of adult inpatients with COVID-19 in Wuhan, China: a retrospective cohort study. Lancet 395(10229):1054-1062. https://doi.org/10.1016/S0140 $-6736(20) 305$

Publisher's Note Springer Nature remains neutral with regard to jurisdictional claims in published maps and institutional affiliations. 